\title{
Plasma lipid concentrations: the concept of "normality" and its implications for detection of high cardiovascular risk
}

\author{
B LEWIS
}

From the Department of Chemical Pathology and Metabolic Disorders, United Medical and Dental Schools of St Thomas's and Guy's Hospitals, London

SUMMARY The relation between serum cholesterol concentrations and the incidence of coronary heart disease is continuous and curvilinear; there is neither epidemiological nor biological evidence to support the existence of a threshold value. There is a clinical need, however, for an acceptable definition of action limits and desirable ranges, based on the evidence that raised cholesterol concentrations are causally related to atherosclerotic heart disease. The European Atherosclerosis Society has proposed a set of cut off points, which, together with age and the presence of other risk factors, direct the clinician to an appropriate level of treatment. Because the changes of serum cholesterol during adult life appear unphysiological, these action limits do not require adjustment for age.

The distribution of serum cholesterol in the United Kingdom population is such that a case finding strategy is required to identify the many persons at very high risk of coronary disease. Measurements of triglyceride, high density lipoprotein, apolipoproteins, and the investigation of hyperlipoproteinaema are informative but less mandatory.

\section{Risk factors as causes}

Much epidemiological research on coronary heart disease (CHD) over the past 30 years has been directed to identifying characteristics such as habits and biometric and laboratory variables that are related to the probability of developing overt CHD. The thrust of these studies has been to define predictive associations between such characteristics and the risk of CHD. ${ }^{1}$ A good deal of discussion has centred around whether such associations reflect aetiology and, if so, whether reduction of risk factors is followed by reduction in incidence of CHD. Examples of non-causal associations abound. Ischaemic ECG changes are predictive of a high risk of CHD merely because they reflect existing CHD. Presence of a diagonal ear lobe crease is associated with a $50 \%$ increase in the risk of CHD, but few would argue that this reflects causality or that judicious plastic surgery might lessen their risk. An inverse relation between high density lipoprotein cholesterol concentration and risk of CHD has often been reported, ${ }^{2}$ though less consistently than other risk factors ${ }^{34}$; but at present there is little evidence that reflects a directly protective role of this lipoprotein.
Several characteristics of associations are necessary to their causal interpretation, including temporal sequence (the putative cause must precede the disease), strength, and presence of a dose response relation, and independence - that is, that it should not merely reflect a correlation with some other causally important variable and consistency. ${ }^{5}$ If the epidemiology is consistent with information from other disciplines the body of evidence must be particularly cogent, and then justifies putting the aetiological theory into clinical practice.

These criteria of causality have been abundantly fulfilled for the relation between serum cholesterol (or low density lipoprotein cholesterol) concentrations and CHD. ${ }^{6}$ Not only do the epidemiological data have the characteristics listed, but they agree with several other sources of information. Clinically, an eight-fold increase in CHD has been observed in patients with familial hypercholesterolaemia, ${ }^{7}$ a genetic disorder of lipoprotein metabolism probably affecting about 120000 persons in the United Kingdom. A high prevalence of CHD and of peripheral atherosclerosis is also evident in remnant (type III) hyperlipoproteinaemia. ${ }^{89}$

In controlled trials of serum cholesterol reduction a 
powerful association has been shown between the change in serum cholesterol concentration and the reduction in CHD. ${ }^{10}$ Atherosclerosis develops in two distinct strains of animals with inherited hyperlipidaemia $^{1112}$; lesions similar to atherosclerosis arising in animals with hyperlipidaemia induced by diet show partial regression when cholesterol concentrations are reduced. ${ }^{13}$ Finally, much is known about the mechanisms by which low density lipoproteins interact with elements of the arterial intima in ways that would influence atherogenesis.

For the relation between serum triglyceride and $\mathrm{CHD}$, and that between high density lipoprotein cholesterol and CHD, the evidence is less compelling. These are profoundly important areas for research, and some provisional clinical standpoint is necessary when patients present with hypertriglyceridaemia of varying degrees. The relation between triglyceride and CHD has been found in two recent studies ${ }^{445}$ to be independent of other risk related variables - that is, high density lipoprotein cholesterol and obesitybut in some studies evidence of a dose response relation is lacking. Some disorders associated with severe hypertriglyceridaemia seem not to be associated with increased risk of $\mathrm{CHD},{ }^{8}$ unlike familial combined hyperlipidaemia, presenting with more moderate hypertriglyceridaemia accompanied by high risk of CHD. ${ }^{8}$ There is no evidence from rigorous controlled trials, and little or none from animal models, to establish a causal link between hypertriglyceridaemia and CHD. Nevertheless, a strong clinical suspicion remains that this relation is a real one, based largely on the prevalence of raised triglyceride concentrations in patients with CHD in case control studies. ${ }^{14}$ Future research may clarify whether certain lipoproteins among the wide range of plasma particles rich in triglycerides are selectively related to atherogenesis, such as the denser, smaller "remnant" lipoproteins, and are also related to thrombotic tendency.

Many longitudinal studies have shown that the relation between high density lipoprotein cholesterol and risk of CHD is strong and independent. It is not entirely consistent, however, as no significant independent predictive power was found in two large scale studies. ${ }^{34}$ Nor is there affirmative evidence from controlled trials. Progression of atherosclerosis was not significantly related to high density lipoprotein cholesterol in one controlled angiographic trial of lipid reduction, ${ }^{15}$ though a strong relation to low density lipoprotein cholesterol was evident. In two other angiographic trials, however, evolution of disease was related to concentrations of high density lipoprotein cholesterol and to total cholesterol. ${ }^{1617}$ In relation to mechanisms by which high density lipoprotein could influence the pathogenesis of atherosclerosis, there is impressive evidence that the $\mathrm{HDL}_{3}$ subclass serves as an acceptor of free cholesterol from cells, so initiating the putative mechanisms of reverse transport of cholesterol from peripheral tissues to the liver. Nevertheless, there is insufficient evidence to suggest that variation in the plasma concentrations of high density lipoprotein or any of its components within their usually observed ranges influences the rate of reverse transport; these concentrations are not independently related to the size of the measurable tissue pools of cholesterol. ${ }^{18}$ Certain genetic disorders associated with extremely low concentrations of high density lipoprotein components are accompanied by a familial aggregation of CHD, ${ }^{19}$ but in other such disorders (Tangier disease, fish-eye disease, and presence of apo A-I (Milano), there is little or no excess risk of coronary disease.

In summary, there is convincing evidence that raised plasma concentrations of low density lipoprotein have a causal role in atherosclerotic heart disease; but the clinical importance of plasma triglyceride and high density lipoprotein cholesterol concentrations requires considerable further evaluation.

\section{Liproprotein risk factors as continuous variables}

The clinical and preventive medicine approaches to CHD risk factors are profoundly influenced by the distribution and prevalence of these variables in the population. A discontinuous relation between the variable and its associated risk might suggest a "natural" cut off point separating the range accompanied by risk from the range not so associated. Those epidemiological studies with sufficient statistical emphasis, however, have shown continuous linear or curvilinear correlations - that is, there has been no indication of a threshold separating a high risk range from a range accompanied by low or zero risk. This seems to hold for blood pressure in relation to CHD and stroke, for smoking in relation to several associated diseases, and for serum cholesterol concentration. Throughout the range of serum cholesterol concentrations seen in countries in which CHD is common, each increased from the lowest quintile of the distribution to the highest is accompanied by a greater risk of CHD. This is evident in the uniquely large $(n=360000)$ longitudinal study of men screened for entry into the Multiple Risk Factor Intervention trial. ${ }^{20}$ Other studies in which a similarly continuous relation has been observed between serum cholesterol and incidence of CHD are the longitudinal phase of the Seven Countries study, the Framingham study, the Whitehall study and the Hawaiian Heart study.

By contrast, risk of CHD was not higher in the 


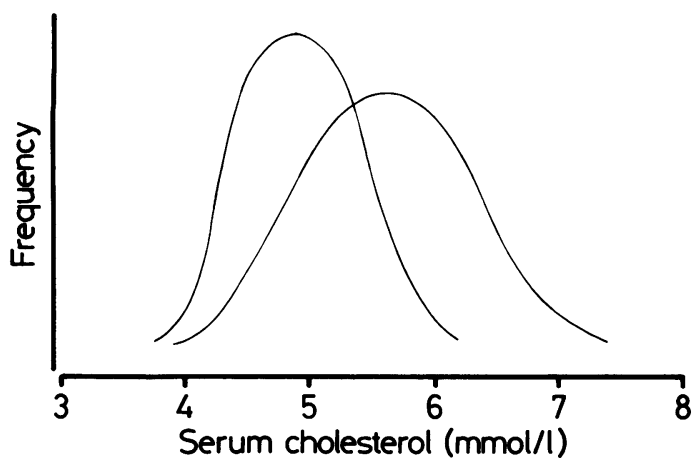

Figure Present distribution of plasma cholesterol concentration in British men and women (right hand curve) and the predicted effect of dietary changes on this distribution (left hand curve).

second quintile of serum cholesterol than that in the lowest quintile in the United States Pooling Project, the Stockholm Prospective Survey, and the Israeli Heart Study.

The lowest quintile of serum cholesterol in persons studied in the Multiple Risk Factor International Trial, the Seven Countries study, and the Framingham study comprised those with cholesterol concentrations less than $4-4.7 \mathrm{mmol} / \mathrm{l}$, implying that values exceeding this range are associated with greater lipoprotein mediated risk of CHD. This conclusion agrees with observations in cross cultural studies: when mortality from CHD and mean cholesterol concentrations were compared in several population groups in the Seven Countries study ${ }^{21}$ an even wider, linear, relation was evident. In the International Atherosclerosis Project ${ }^{22}$ the extent of coronary atherosclerosis from 17 widely disparate populations showed a rank order congruent with that of mean serum cholesterol.

Within the range of cholesterol concentrations commonly seen in populations at high risk $(4-8 \mathrm{mmol} / \mathrm{l})$, therefore, there is no good evidence of a threshold for the serum cholesterol-CHD risk correlation; instead a continuous, usually curvilinear, gradation of risk with increasing serum cholesterol is seen. This epidemiological conclusion is consistent with what is known of the interactions between lipoproteins and the components of the arterial intima; there is no suggestion from this source that existence of a threshold is biologically plausible.

The population strategy for reduction of CHD in the community is consistent with this concept that the serum cholesterol-CHD correlation is continuous over a wide range. Using educational and other mea- sures directed to the population as a whole, the population strategy aims to change the distribution of risk related variables (serum cholesterol, smoking, blood pressure, body weight) towards lower risk. In the context of serum cholesterol distribution the goal is to recommend a gradual but sufficient change in national dietary patterns to reduce the modal cholesterol concentration of countries such as the United Kingdom by $10-15 \%$ - that is, to that of countries with a lower rate of CHD. As the reduction in those subjects with concentrations in the upper part of the distribution will be greater than the mean reduction, and the reduction in those with initially low serum concentrations will be less than the mean reduction, ${ }^{23}$ the effect will be to produce a narrower distribution shifted to the left (figure). If the cholesterol-CHD correlation is curvilinear it follows that the greatest reduction will be among those with serum concentrations in the upper part of the distribution where the relation to risk of CHD is steepest.

For those with serum cholesterol concentrations conferring increased risk of CHD, these considerations of the form of the cholesterol risk relation have profound implications for the concepts of normal or reference ranges for serum cholesterol concentration, and of action limits.

\section{“Normal" serum cholesterol concentrations}

Serum cholesterol concentration has, essentially, unimodal distribution, though a few people have extremely high concentrations resulting from major genetic hyperlipidaemias.

For no other analyte commonly measured by chemical pathologists has there been greater diversity of views concerning reference ranges and optimal distributions than for serum cholesterol concentration. Upper values for reference ranges such as $7.8 \mathrm{mmol} / 1$ are used by several laboratories, and higher values are by no means unknown. These have been variously based on established statistical criteria such as limits set two standard deviations above the mean of an apparently healthy population sample, on the 90th centile for young adults, or on more arbitrary choice $(7.8 \mathrm{mmol} / \mathrm{l}$ corresponding to the convenient round value $300 \mathrm{mg} / \mathrm{l}$ ). Some arcane reasons have been put forward when selecting bases for deriving such limits, of which the most irrational and potentially harmful is the wish to avoid assigning too many people to a hypercholesterolaemic range.

Several problems attend the use of statistically derived ranges based on measurements on a sample from a particular normal population. The most conspicuous is the wide variation between the distribution seen in healthy people in different populations; it has been pointed out that the distributions of some 
normal populations barely overlap. ${ }^{24}$ Who, then, is "normal"? A person with a serum concentration of, say, $6 \mathrm{mmol} / \mathrm{l}$ would be regarded as normal in the United Kingdom but would be given treatment in Italy or Japan. No generally applicable "normal range" can be derived from such observations.

A further problem requiring discussion is the relation of serum cholesterol and low density liproprotein cholesterol concentrations to age. In neonates mean concentrations are of the order of $2 \mathrm{mmol} / \mathrm{l}$, and there is a steep increase in the first four or five years of life. There is little further change in childhood and early adult life. In Western countries with high rates of CHD, mean concentrations of cholesterol in adults tend to increase from the fourth decade to reach a peak in the sixth decade, subsequently decreasing in later life. ${ }^{25}$ For this reason age adjusted ranges are sometimes provided to take into account the increase in middle age. Interestingly, this trend with adult age is common to other risk related variables such as blood pressure and body weight in populations prone to CHD.

If the rise of mean serum cholesterol in middle life is physiological it should be seen in all populations. This is not the case, however: in several studies of populations with a low incidence of CHD, plasma cholesterol $^{2627}$ and triglyceride ${ }^{28}$ showed no increase during adult life. It is no longer regarded as appropriate to adjust desirable ranges of body weight or blood pressure for age. Similarly, the rise of serum cholesterol in middle age is best regarded as unphysiological, reflecting an increased prevalence of hyperlipidaemia in the fifth and sixth decades of life. The decreasing lipid values seen in cross sectional studies in the seventh and eighth decades may also be unphysiological, as they reflect (at least in part) a selectively greater mortality from cardiovascular disease among people with concentrations in the upper part of the distribution.

Sex differences are seen in serum cholesterol concentrations and, to a more pronounced extent, low density liproprotein cholesterol concentrations ${ }^{25}$; in premenopausal women these values are lower than in men of similar age, and following a steep perimenopausal increase they are subsequently higher in women.

Differences have been shown in the distribution of at least one genetically determined factor that is known to influence serum cholesterol concentration. Genetic polymorphism of apolipoprotein $\mathrm{E}$ results in the existence of six phenotypes: E2/2, E2/3, E3/3, $E 3 / 4, E 2 / 4$ and E4/4; the E3/3 phenotype is the commonest. Apo E2/2 is associated with lower than average serum cholesterol, and apo E3/4 and apo E4/4 with higher than average. ${ }^{29}$ While in theory differences between populations in distribution of serum cholesterol may have a genetic component, there is evidence that environmental factors play the major part: studies of migrating populations have shown that the serum cholesterol distribution of the migrant group rapidly approaches that of the host population-for example, Japanese in Hawaii and California, Yemenites in Israel. In an institutional crossover nutritional experiment the large difference in mean serum cholesterol concentrations between white and black South Africans was reversed by exchanging their diets in a six month study. ${ }^{30}$

\section{Desirable serum cholesterol concentrations, and the selection of action limits}

Several reasons militate against the use of the usual statistical bases for defining a normal range of serum cholesterol. An alternative approach is to identify serum concentrations that are associated with minimum risk of death or disease. This concept is the basis of the definition of "ideal" body weight, and current definitions of diabetes mellitus are based on concentrations of blood glucose associated with the appearance of microangiopathy. ${ }^{31}$ The direct relation between risk of CHD and serum cholesterol provides the only rational basis for recognition of a desirable cholesterol concentration. (Serum cholesterol is also positively associated with the incidence of atherosclerosis affecting the lower limbs). Conversely, there is no evidence that low serum cholesterol is causally associated with disease, ${ }^{32} 33$ though extremely low concentrations are markers for abetalipoiveproteinaemia and Tangier disease, two extremely rare, serious genetic disorders of apolipoprotein B and apo-A-I metabolism, respectively.

The issue of desirable cholesterol concentrations, in relation to the risk of CHD, has been repeatedly addressed in recent years. A multidisciplinary workshop proposed that mean concentrations for adult populations should optimally be about $4 \mathrm{mmol} / \mathrm{l}$ and, more practicably, $4.7 \mathrm{mmol} / \mathrm{I}^{24}$; adding one standard deviation would result in an upper limit for the individual of about $5-5.7 \mathrm{mmol} / \mathrm{l}$. A World Health Organisation expert committee recommended that desirable mean concentrations should be less than $5.2 \mathrm{mmol} / 1^{34}$; the committee did not address the issue of individual concentrations. At a National Institute of Health consensus development conference ${ }^{35}$ cut off points demarcating moderate and high risk categories were set at the 75th and 90th centiles respectively, for the American population: $>5.2 \mathrm{mmol} / \mathrm{l}$ and $>5.7 \mathrm{mmol} / 1$ for age $20-29 ;>5.7$ and $>6.2 \mathrm{mmol} / 1$ for age $30-39$; and $>6.2$ and $>6.7 \mathrm{mmol} / \mathrm{l}$ for age 40 and over. In discussing the goals of population strategy and individual care, the National Institute of Health panel regarded $4.7 \mathrm{mmol} / 1$ as an attainable 
goal for adults up to age 30 , and $5 \cdot 2 \mathrm{mmol} / \mathrm{l}$ as a goal for those above this age.

Most recently the study group of the European Atherosclerosis Society reported its conclusions, ${ }^{36}$ discussing maximum limits for serum cholesterol in considerable depth. The option of using quartiles of the cholesterol distribution was rejected as this approach would have led to international differences in cut off points. The Society expressed the view that, "the interaction between lipoproteins and the arterial wall is presumably a function of their plasma concentrations, favouring the selection of absolute levels".

It is necessary to emphasise, as the European Atherosclerosis Society study group repeatedly does, that absolute action limits must be used flexibly and regarded only as guidelines. Management should be that of the person as a whole, and should not be based on the laboratory finding alone. Hence the physician must take into account the individual's age, the presence, severity, and duration of other CHD risk factors such as hypertension, diabetes, and smoking, the presence and strength of a family history of CHD, and the age of its presentation in relatives.

Given the continuous nature of the relation between incidence of CHD and levels of metabolic and most other risk factors, the selection of action limits and definition of desirable serum values is necessarily arbitrary. Provision of action limits is for convenience in clinical management, and their use must be modulated by overall risk, age, and family history.
Treatment for hyperlipidaemia is also influenced by cardiovascular condition; in particular, meticulous control is appropriate in patients who undergo revascularisation procedures such as coronary artery bypass grafting.

Table 1 is derived from the guidelines suggested in the European Atherosclerosis Society statement on the prevention of CHD. ${ }^{36}$ Certain of the qualifications in the second column of these guidelines have been referred to, and others require brief comment. One is that all modifiable risk factors require attention, as the effects of hyperlipidaemia, hypertension, smoking and diabetes on risk of CHD are strongly cumulative. Secondly, the intensity of treatment - that is, the degree of dietary change, the administration of drugs (and the type, dose, and number of drugs used) - is graded in relation to the severity of the lipid abnormality and the overall risk level. The extent of treatment is also influenced by age: the value of risk factor reduction is clearly greatest in younger patients, in whom, it is hoped, atherosclerosis may be prevented or delayed, and growth of existing plaques be retarded or arrested at an earlier stage. Conversely, less intensive treatment and lesser degrees of lipid reduction may be appropriate in older people, even though it is unreasonable to select an arbitrary age beyond which treatment should be withheld.

In summary, the risk of CHD becomes progressively greater when serum cholesterol concen

Table 1 Assessment and management of the hyperlipidaemic patient

\begin{tabular}{|c|c|c|}
\hline Concentration ( $\mathrm{mmol} / \mathrm{l})$ & Investigation and assessment & Management \\
\hline $\begin{array}{l}\text { Cholesterol 5.2-6.5 } \\
\text { Glyceride }<2 \cdot 3\end{array}$ & $\begin{array}{l}\text { Take into account age, family history } \\
\text { of } \mathrm{CHD} \text {, blood pressure, smoking, } \\
\text { high density lipoprotein cholesterol } \\
<1 \mathrm{mmol} / 1\end{array}$ & $\begin{array}{l}\text { Emphasise general dietary guidelines for serum } \\
\text { lipid reduction; deal with associated risk factors } \\
\text { and overweight }\end{array}$ \\
\hline $\begin{array}{l}\text { Cholesterol 6.5-7.7 } \\
\text { Glyceride }<2 \cdot 3\end{array}$ & $\begin{array}{l}\text { Take into account age, family history } \\
\text { of CHD, blood pressure, smoking, } \\
\text { high density lipoprotein cholesterol } \\
<1 \mathrm{mmol} / \mathrm{l}\end{array}$ & $\begin{array}{l}\text { (i) Prescribe lipid lowering diet, restricting } \\
\text { food energy if overweight } \\
\text { (ii) Monitor response; promote compliance } \\
\text { (iii) Consider lipid lowering drug if cholesterol } \\
\text { persistently high }\end{array}$ \\
\hline $\begin{array}{l}\text { Cholesterol }<5.2 \\
\text { Glyceride } 2 \cdot 3-5 \cdot 6\end{array}$ & $\begin{array}{l}\text { Identify possible causes of } \\
\text { hypertriglyceridaemia: alcohol abuse, } \\
\text { obesity, diabetes, use of diuretics, } \\
\text { oestrogens, retinoids }\end{array}$ & $\begin{array}{l}\text { Restrict food energy if overweight, and emphasise } \\
\text { general dietary guidelines for lipid reduction; } \\
\text { deal with underlying cause if possible }\end{array}$ \\
\hline $\begin{array}{l}\text { Cholesterol } 5 \cdot 2-7 \cdot 8 \\
\text { Glyceride } 2 \cdot 3-5 \cdot 6\end{array}$ & $\begin{array}{l}\text { Take into account age, family history } \\
\text { of CHD, blood pressure, smoking, } \\
\text { high density lipoprotein cholesterol } \\
<1 \text { mmol/l; identify possible cause of } \\
\text { hypertriglyceraemia }\end{array}$ & $\begin{array}{l}\text { (i) Prescribe lipid lowering diet, restricting } \\
\text { food energy if overweight } \\
\text { (ii) Monitor response; promote compliance } \\
\text { (iii) Consider lipid lowering drug if cholesterol } \\
\text { permanently high } \\
\text { Restrict food energy if overweight, and emphasise } \\
\text { general dietary guidelines for lipid reduction; } \\
\text { deal with underlying causes if possible }\end{array}$ \\
\hline $\begin{array}{l}\text { Cholesterol }>7.8 \\
\text { or } \\
\text { Glyceride }>5 \cdot 6 \text {, or both }\end{array}$ & $\begin{array}{l}\text { Assess cardiovascular condition and } \\
\text { investigate fully for causes of secondary } \\
\text { hyperlipidaemia }\end{array}$ & $\begin{array}{l}\text { Proceed as above or consider referral to } \\
\text { specialised lipid clinic; treatment with } \\
\text { appropriate drug(s) is commonly necessary. }\end{array}$ \\
\hline
\end{tabular}

Glyceride $>5 \cdot 6$, or both investigate fully for causes of secondary hyperlipidaemia 
trations exceed the range $4 \cdot 7-5 \cdot 2 \mathrm{mmol} / 1$, and those people with serum values exceeding $5 \cdot 2 \mathrm{mmol} / 1$ should be considered for some degree of lipid reduction treatment. For most such people, in particular those with serum concentrations in the range 5.2 to $6.5 \mathrm{mmol} / \mathrm{l}$, management amounts to reinforcement of existing recommendations made to the population as a whole, comprising healthy eating, weight control, avoidance of smoking, and suitable regular exercise. The concept of a "normal range" of serum cholesterol is untenable. In its place the continuous nature of the relation between cholesterol concentration and risk of CHD should be understood, and a series of flexibly interpreted action limits may be offered as guidelines to the several levels of possible intervention. Most simply, it may be indicated on a pathology report and elsewhere that the risk of CHD increases progressively with serum cholesterol concentrations exceeding $5 \mathrm{mmol} / \mathrm{l}$.

\section{Serum triglyceride and high density lipoprotein cholesterol}

Current evidence relating serum triglyceride risk to CHD was summarised in an earlier section. The incompleteness of this evidence precludes any objectively based recommendations analogous to those for serum cholesterol. When hypertriglyceridaemia is a manifestation of remnant (type III) hyperlipoproteinaemia or familial combined hyperlipidaemia, treatment for these disorders is justified by the evidence that they are associated with an excess risk of CHD. The 95th centile for serum triglyceride in men and women aged 25-59 in the United Kingdom Risk Factor Prevalence study in fasting serum samples was $3.8 \mathrm{mmol} / \mathrm{l}$ (and in random samples it is about $4.7 \mathrm{mmol} / \mathrm{l}$ ) (Guyer BM, Lewis B, Minn JL, et al. Abstract presented at VIIth International Symposium on Atherosclerosis, Melbourne, 1985.) The 90th centile, in the fasted state, was $2.7 \mathrm{mmol} / \mathrm{l}$. There is no sufficiently strong evidence to justify recommendations influencing treatment that values in the range 3-5 $\mathrm{mmol} / \mathrm{l}$ are a causal factor for CHD. Nevertheless, concentrations in and above this range are often associated with obesity, alcohol abuse, and diabetes. Such disorders justify treatment in their own right, and their management will commonly lead to a reduction in serum triglyceride. Secondly, the fat modified diet reduces serum triglyceride, and as this diet is already a central component of the population strategy for reduction of CHD, it may be particularly recommended to those with mild to moderate hypertriglyceridaemia.

Triglyceride concentrations in the range 5-11 mmol/l are relatively uncommon. The National Institute of Health consensus conference on treat- ment of hypertriglyceridaemia considered that treatment should be given for concentrations of this magnitude, in view of the possible risk of $\mathrm{CHD} .^{37}$ This is a reasoned interim standpoint supported by recent evidence: ${ }^{3839}$ it is prudent to treat such patients, especially if, as is common, they have a strong family history of CHD. When serum triglyceride exceeds $11 \mathrm{mmol} / \mathrm{l}$ in the fasted state, and particularly when it exceeds $20 \mathrm{mmol} / \mathrm{l}$, the risk of recurrent acute pancreatitis is an unequivocal justification for treatment. ${ }^{378}$

In part, the interpretation of high density lipoprotein cholesterol concentrations follows similar lines. The dearth of information on the possibility that this lipoprotein has a direct protective role against CHD has already been referred to in this review. Statistical reference ranges for British adults (10th and 90th percentiles) are 0.8 to $2.0 \mathrm{mmol} / 1$ in men, and 1.2 to $2.2 \mathrm{mmol} / \mathrm{l}$ in women. ${ }^{25}$ Low concentrations of high density lipoprotein cholesterol are often seen in obesity, and are slightly but significantly reduced in cigarette smokers: treatment and counselling are, of course, indicated.

\section{The distribution of serum cholesterol concentrations in Britain}

In an interim report on over 3000 men and women aged 25-59 years (Guyer BM, Lewis B, Minn JL, et al. Abstract presented at VIIth International Symposium on Atherosclerosis, Melbourne, 1985.) the distribution of serum cholesterol concentration was slightly skewed to the right, with a mean of $5.7 \mathrm{mmol} / 1$. Of this population sample, $23 \%$ had cholesterol concentrations exceeding $6.5 \mathrm{mmol} / \mathrm{l}$. This is associated with a mortality from CHD about twice as great as that of people with concentrations of less than $5 \mathrm{mmol} / \mathrm{l}$. Values exceeding $5.2 \mathrm{mmol} / \mathrm{l}$ were found in $63 \%$ of the population.

These prevalences are higher than those in the United States of America and most other countries. In the United States of America, mean serum cholesterol concentration is believed to have fallen by $3-8 \%$ over the past two decades, ${ }^{40}$ a change that occurred at the same time as a striking decline in mortality from CHD. It can be anticipated that a similar trend in serum cholesterol distribution will take place in Britain, or may already be happening. In the age groups 30-39 and 40-49 mortality from CHD in England and Wales has consistently fallen in the years reported since 1978. Patterns of food selection are changing in the United Kingdom, and public awareness of recent nutritional guidelines by the Department of Health and Social Security Committee on medical aspects of food policy panel and by the National Advisory Council on Nutrition Education is 
likely to accelerate this trend.

It may reasonably be expected, therefore, that the prevalence of undesirable concentrations of serum cholesterol in Britain will decrease over the next several years. We have attempted to estimate the extent of this change, based on various sets of assumptions as to trends in consumption of saturated and polyunsaturated fatty acids, cholesterol, and fibre. ${ }^{41}$ These suggest that the incidence of cholesterol concentrations of $>6.5 \mathrm{mmol} / \mathrm{l}$ may decrease to between 4 and $10 \%$ in the young and middle aged.

These estimates of prevalence of hypercholesterolaemia have substantial implications for pathologists and physicians working in the detection and management of lipoprotein disorders.

\section{Individual and population strategy for reduction of CHD}

The population approach to risk reduction aims to improve health related behaviour in the community as a whole. This is an essential component of preventive medicine policy, deserving endorsement by doctors in interactions with patients and the public at all times. The individual approach seeks to identify those at particularly high risk, with a view to management and follow up in a clinical setting. These are complementary strategies, and in no sense alternatives. Only the former strategy could reach the large proportion of the population with moderate risk factors who are at risk of CHD. Educational and other measures comprising the population strategy, however, are inadequate for those at high risk. Familial hypercholesterolaemia and remnant hyperlipoproteinaemia, which together probably affect some 120000 to 140000 people in the United Kingdom seldom respond to the moderate dietary change advocated for the population; most patients require treatment with drugs to achieve adequate reduction in lipids.

Purely clinical aspects of the individual strategy are beyond the scope of this review. For the pathologist, pertinent aspects include the diagnosis of lipoprotein disorders and the provision of laboratory services for growing numbers of specialised lipid clinics.

\section{Detection and diagnosis of hyperlipidaemia}

Occasionally, hyperlipidaemia is diagnosed clinically, when the presence of xanthomas is noted by the general physician, orthopaedic surgeon, or dermatologist. But most hyperlipidaemic patients do not have xanthomas; at least one third of patients, even those with severe familial hyperlipidaemia, do not show specific physical signs. For practical purposes, then, detection of hyperlipidaemia depends on mea- surement of serum lipid concentration.

But for whom should lipid measurements be provided? If it is accepted that detection, management, and follow up of substantial degrees of hyperlipidaemia are necessary to the individual strategy for the prevention of CHD, three optional approaches to the recognition of affected individuals may be considered.

\section{(i) GENERAL SCREENING}

The public may be offered direct access to risk factor assessment, including blood pressure measurement and lipid analyses. This has been adopted in Norway, and is available on a fee for service basis, or as part of an employment benefit package in many countries. It must be emphasised that this approach, like other diagnostic procedures, carries with it the obligation to provide treatment and systematic follow up facilities. The future of general screening is therefore linked with increased availability of such facilities. At present there are persuasive arguments against the provision of general lipid screening on the National Health Service. There are considerable cost implications, and the case has not been made for diverting resources from other services. If general screening were made available in the near future the large case load generated would overload the specialised facilities for management currently available. At preseng there are only 74 such clinics in the United Kingdone?

(ii) SELECTIVE SCREENING

It is possible to identify the subsets in the population in whom CHD risk factors are overrepresented. By directing risk factor assessment to those subsets, measurements can be confined to those likely to have abnormal findings. In many instances such persons are at high cardiovascular risk on other grounds, hence detection of lipid risk factors is of particular importance to them. There is, therefore, a strong case for risk factor assessment in the following categories:

(i) Those giving a positive family history of CHD, particularly when this is of early onset, and when several relatives are affected.

(ii) Those with definite or suspected xanthomas.

(iii) Those in whom corneal arcus or xanthelasmas are detected before 40 years of age.

(iv) Those with a family history of hyperlipidaemia.

(v) Diabetic patients.

(vi) Hypertensive patients.

(vii) Obese patients.

Assessment of risk factors is of potential benefit to people in these categories; on the other hand, many people with moderate hypercholesterolaemia would 0 not be identified if lipid measurements were confined $\overparen{D}$ to these groups: $36 \%$ of the British population have a $\stackrel{\mathcal{\infty}}{+}$ positive family history of CHD in a first degree rela- 
tive; examination of such people would lead to detection of $43 \%$ of those with cholesterol concentrations of $>6.5 \mathrm{mmol} / \mathrm{l}$ (Lewis B, Mann JL, Rose LB, et al., unpublished observations). Other proposed criteria for selective screening are of the same order of sensitivity. Were all such criteria used, some two thirds of the population would undergo assessment; yet one third of all people with serum cholesterol of $>6.5 \mathrm{mmol} / 1$ would not have been examined. Hence selective screening, however extensive, would miss a considerable proportion of people with moderate or severe hyperlipidaemia.

\section{(iii) CASE FINDING}

During a full clinical examination for an intercurrent medical problem, it is normal practice to take a smoking history, record body weight, measure blood pressure, and test for glycosuria. The examination therefore provides information on several cardiovascular risk factors. It is sound and established practice to act on this information-that is, to introduce a component of preventive medicine into such a consultation. Furthermore, blood analyses are commonly requested-for example, haematological and biochemical profiles, or specific tests such as those of thyroid function.

The addition of serum cholesterol measurement to such investigations permits comprehensive assessment of risk of CHD. Given the importance of detecting and dealing with hypercholesterolaemia, the value of this investigation is probably greater than many that are routinely included in the "biochemical profile" without specific indications. Thus the case finding approach to recognition of hyperlipidaemia lacks the drawbacks of general screening and has two advantages. One is that the effect of laboratory workload will be modest compared with that of general screening. If lipid measurements are performed when a patient is first seen and is fully examined by a general practitioner, or consultant physician, or cardiologist, the number of additional analyses per year will be comparatively modest; yet over several years a large proportion of the population will be tested. Secondly, the measurement is performed within the context of the doctor-patient relationship, which has implications for the level of understanding and motivation of the patient, for the provision of management within a clinical setting, and for follow up.

This approach is complementary to selective screening, the two strategies affording the best chance of detecting major hyperlipidaemia in the population, without the financial implications of general screening for the laboratory and for staffing.

Recent progress in dry chemistry methods and improvement in reflective meter design are likely to have an impact on the diagnosis and monitoring of hyper- lipidaemia, which at least will be as great as it has been on diabetic care. Using fingerprick samples, and in some instances accepting whole blood, the instruments produce results within three minutes at a highly competitive unit cost. Some have had teething troubles, but there is little doubt that accuracy is possible. The convenience to doctor and patient is obvious. The workload generated by case finding of hypercholesterolaemia will probably be shared between this approach and central laboratories.

When pronounced hyperlipidaemia is detected, it is often preferable that the patient be investigated and treatment initiated in a specialised unit. Several of these are staffed partly or entirely by chemical pathologists with postgraduate clinical training.

\section{Investigation of lipoprotein disorders associated with high risk of CHD}

It is evident that accurate measurement of serum cholesterol concentration is the single most important biochemical investigation in assessment of risk of CHD. It is, however, possible to undertake a far more extensive test repertoire, including triglyceride and high density lipoprotein cholesterol concentration, direct measurement of low density lipoprotein cholesterol, full lipoprotein fractionation, including very low density lipoprotein lipid measurement and separation of two main subclasses of high density lipoprotein. Assays of apo B in serum and in low density liproprotein, and of serum apo A-I, are also available. Some of this range of investigations are essential in classifying and understanding major disorders of lipoprotein metabolism. To assess the risk of CHD in large numbers of people, on the other hand, cost implications alone argue for some austerity in the test repertoire; each investigation should clearly contribute to decisions on the need for and type of treatment. In addition, measurement of triglyceride and triglyceride rich lipoproteins is conventionally carried out in the fasted state to avoid the potentially wide fluctuations that occur during alimentary lipaemia. This usually necessitates a second visit by the patient.

I have already referred to some of the present limitations of the evidence linking CHD with triglyceride and high density lipoprotein cholesterol concentrations. A conventional view has been that triglyceride measurement does not add to the prediction of risk of CHD if the cholesterol concentration is known. As the classification of hyperlipidaemic states requires knowledge of cholesterol and triglyceride concentrations, one suggested approach has been to measure cholesterol alone, and to recall people with cholesterol concentrations exceeding a chosen cut off value for a further cholesterol measurement, together with triglyceride and high density lipoprotein choles- 
terol in the fasted state. Cholesterol concentrations of $5.2 \mathrm{mmol} / \mathrm{l}$ and $6.5 \mathrm{mmol} / \mathrm{l}$ are recommended as cut off points; if this approach is adopted the latter value seems preferable.

If the patient has definite or suspected xanthomata, if other grounds are present for suspecting presence of a major metabolic disorder, or if the serum is noticeably lactescent, a sample should certainly be obtained in the fasted state for cholesterol and triglyceride measurements. One justification for inclusion of high density lipoprotein cholesterol measurement in the assessment of risk factor is the occurrence of mild hypercholesterolaemia (7-7.5 $\mathrm{mmol} / \mathrm{l}$ ) due to unusually high concentrations of high density lipoprotein, with normal concentrations of other lipoproteins. As treatment is not indicated there is an advantage in recognising this uncommon entity of high density lipoprotein hyperpoproteinaemia. The wider question that remains concerns the value of measuring high density lipoprotein cholesterol in adding to the prediction of risk. Considerable further research is needed before this can be clearly settled; for the present, measurement of high density lipoprotein cholesterol should be regarded as of uncertain clinical value. Apart from high density lipoprotein related hyperlipidaemia, there is no rigorous proof that knowledge of high density lipoprotein cholesterol should currently influence therapeutic decisions.

Considerable interest has been stimulated by case control studies, suggesting that concentrations of apolipoproteins, in particular apo $\mathrm{B}^{\mathbf{4 2}}$ and possibly also apo $\mathrm{A}-\mathrm{I},{ }^{43}$ discriminate better than lipid concentrations between CHD patients and controls. There is as yet little confirmation of this from longitudinal studies, and little or no exploration of possible implications for treatment. But the topic is undoubtedly a growth area in lipoprotein research and may well earn a place in clinical assessment of risk of CHD.

In the Green Paper on primary health care, criteria for high standards of practice are proposed, including the provision of preventive activities. ${ }^{44}$ Prevention of CHD is referred to specifically. Established CHD is largely a cardiological problem; but its prevention will require the combined efforts of several disciplines, with the general practitioner very much at the forefront. The chemical pathologist has an important and potentially rewarding analytical and interpretative role.

\section{References}

1 Stamler J. Lifestyles, major risk factors, proof and public policy. Circulation 1978;58:3-19.

2 Gordon T, Kannel WB, Castelli WB, Dawber TR. Lipoproteins cardiovascular disease and death. The Framingham Study. Arch Intern Med 1981;141:1128-31.

3 Wiklund O, Wilhelmsen L, Ehnveldt D, Wedel H, Valek J,
Gustafson M. Alpha lipoprotein cholesterol concentration in relation to subsequent myocardial infarction in hypercholesterolemic men. Atherosclerosis 1980;37:47-53.

4 Pocock SJ, Shaper AG, Phillips AN, Walker M, Whitehead TP. High density lipoprotein cholesterol is not a major risk factor for ischaemic heart disease in British men. $\mathrm{Br}$ Med J 1986;292;515-19.

5 Hill AB. Principles of medical statistics. 8th ed. London: The Lancet, 1967:306-13.

6 Lewis B. The lipoproteins: predictors, protectors and pathogens. Br Med J 1983;287:1161-4.

7 Stone NJ, Levy RI, Frederickson DS, Verter J. Coronary artery disease in 116 kindred with familial type II hyperliproproteinemia. Circulation 1974;49:476-88.

8 Lewis B. The hyperlipidaemias: clinical and laboratory practice. Oxford: Blackwell Scientific Publications, 1976

9 Brewer HB (moderator). Type III hyperlipoproteinemia: diagnosis, molecular defects, pathology and treatment. Ann Intern Med 1983;98:623-40.

10 Peto R, Yusuf S, Collins R. Cholesterol-lowering trial results in their epidemiological context. Circulation 1985;72:451.

11 Watanabe Y. Serial inbreeding of rabbits with hereditary hyperlipidemia (WHHL rabbit). Atherosclerosis 1980:36:261-8.

12 La Ville A, Turner PR, Pittalo R, Martini S, Woolf N, Lewis B Hereditary hyperlipidaemia with atherosclerosis in the rabbit due to overproduction of lipoproteins.Arteriosclerosis 1987;7:105-12.

13 St Clair RW. Atherosclerosis regression in animal modelscurrent concepts of cellular and biochemical mechanisms. Progr Cardiovascular Disease 1983;26:109-30.

14 Lewis B, Chait A, Oakley CM, et al. Serum lipoprotein abnormalities in patients with ischaemic heart disease. $\mathrm{Br}$ Med $J$ 1974;3:489-93.

15 Duffield RGM, Lewis B, Miller NE, Jamieson CW, Brunt JNH Colchester ACF. Treatment of hyperlipidaemia retard progression of symptomatic femoral atherosclerosis. A ran domised controlled trial. Lancet 1983;ii:639-42.

16 Brensike JF, Levy RI, Kelsey SF, et al. Effects of therapy with cholestyramine on progression of coronary atherosclerosis: results of the NHLBI type II coronary intervention study. Circulation 1984;69:313-24.

17 Arntzenius AC, Kromhout D, Barth JD, et al. Diet, lipoproteins and the progression of coronary atherosclerosis. $N$ Engl J Med 1985;312:805-11.

18 Blum CB, Dell RB, Palmer R, Ramakrishnan R, Seplowitz AH, Goodman DS. Relationship of the parameters of body cholesterol metabolism with plasma levels of high density lipoprotein cholesterol and the major high density lipoprotein apoproteins. $J$ Lipid Res 1985;26:1079-86.

19 Schaefer EJ. Clinical, biochemical and genetic features in familial disorders of high density lipoprotein deficiency. Arteriosclerosis 1984;4:303-22.

20 Neaton JD, Keller LH, Wentworth D, Borhani NO. Total and cardiovascular mortality in relation to cigarette smoking, serum cholesterol concentration and diastolic blood pressure among black and white males followed up for five years. Am Heart J 1984;108:759-69.

21 Keys A. Seven countries. Cambridge: Harvard University Press. 1980.

22 McGill MC, Arias-Stella J, Carbounell LM, et al. Genera findings of the International Atherosclerosis Project. Lab Invest 1968;18:465-628.

23 Keys A, Anderson JT, Grande F. Serum cholesterol response to changes in the diet. III. Differences among individuals. Metabolism 1968;14:766-75.

24 Blackburn H, Lewis B, Wissler RW. Conference on the health effects of blood lipids: optimal distributions for populations. Prev Med 1979;8:609-732.

25 Lewis B, Chait A, Wootton IDP, et al. Frequency of risk factors for ischaemic heart-disease in a healthy British population. 
Lancet 1974;i:141-6.

26 Shaper AG, Jones KW. Serum cholesterol, diet and coronary heart disease in Africans and Asians in Uganda. Lancet 1959;ii:534-6.

27 Rossouw JE, van Staden DA, Benade AJS et al. Is it normal for serum cholesterol to rise with age? In: Nestel PJ, Fidge NH, eds. Atherosclerosis VII. Amsterdam: Elsevier, 1985:37-40.

28 Antonis A, Bersohn I. Serum triglyceride levels in South African Europeans and Bantu and in ischaemic heart disease. Lancet 1960;i:998-1000.

29 Utermann G, Vogelberg KH, Steinmetz A, et al. Polymorphism of apolipoprotein E. Clin Genet 1979;15:36-62.

30 Antonis A, Bersohn I. The influence of diet on serum lipids in South African White and Bantu prisoners. Am J Clin Nutr 1962;10:484-99.

31 Keen H, Jarrett RJ, Alberti KGMM. Diabetes mellitus: a new look at diagnostic criteria. Diabetologia 1979;16:283-5.

32 Lewis B, Mancini M. Hypocholesterolaemia-a risk factor? In; Schettler G, Gotto AM, Middelhof G, et al, eds. Atherosclerosis VI. Berlin: Springer Verlag 1982;841-72.

33 International Collaborative Group. Circulating cholesterol level and risk of death from cancer in men aged 40-69 years. JAMA 1982;284:2853-9.

34 WHO Expert Committee. Prevention of coronary heart disease. Technical Report Series 678. Geneva: WHO, 1982.

35 National Institutes of Health Consensus Development Conference Statement. Lowering blood cholesterol to prevent heart disease. Washington DC: US Department of Health and Human Services, 1985.

36 European Atherosclerosis Society. Policy statement on the pre- vention of coronary heart disease. Eur Heart $J$ (In press.)

37 Grundy SM. Treatment of hypertriglyceridemia. NIH Consensus Development Conference Summary. Arteriosclerosis 1984;4:296-301.

38 Cambien F, Gacqueson A, Richard JL, Warner JM, Ducimetiere $P$, Claude JR. Is the level of serum triglyceride a significant predictor of coronary death in normal cholesterolemic subjects? Am J Epidermiol 1986;124:624-31.

39 Castelli WP. The triglyceride issue: a view from Framingham. Am Heart $J$ 1986;112:432-7.

40 Stamler J. Coronary heart disease: doing the "right things". $N$ Engl J Med 1985;312:1053-5.

41 Lewis B, Mann JI, Mancini M. Reducing the risks of coronary heart disease in individuals and in the population. Lancet 1986;i:956-9.

42 Sniderman A, Wolfson C, Teng B, et al. Association of hyperapobetalipoproteinemia with endogenous hypertriglyceridemia and atherosclerosis. Ann Intern Med 1982;97:833-89.

43 Reardon MF, Nestel PJ, Craig IH, Harper RW. Lipoprotein predictors of the severity of coronary artery disease in men and women. Circulation 1985;71:881-8.

44 Primary health care. Department of Health and Social Security. 9771. London, HMSO, 1986.

Requests for reprints to: Professor B Lewis, Department of Chemical Pathology and Metabolic Disorders, UMDS of St Thomas' and Guy's Hospital, St Thomas' Hospital, London SE1 7EH, England. 